\section{Rhabdomyolyse nach Power-Strampeln auf dem Fitness-Rad}

Wer im Fitness-Studio oder in den eigenen vier Wänden auf der Stelle radelt, riskiert zwar keine Verkehrsunfälle. Doch es drohen andere Gefahren - etwa ein akuter Zerfall von Skelettmuskulatur.

F rüher saß man auf dem Heimtrainer, auch Sitzergometer genannt. Heute hat das Radfahren ohne richtiges Fahrrad, ohne Fortbewegungseffekt und umgeben von vier Wänden schnittigere Bezeichnungen: Indoorcycling, Powercycling oder Spinning. Betrieben wird diese Art der körperlichen Ertüchtigung oft bis an den Rand der Erschöpfung und immer zu dem Zweck, kardiorespiratorisch und muskulär fitter zu werden.

Sicher ist es nicht beabsichtigt, so lange zu strampeln, bis sich die Muskeln auflösen. Dennoch kommt genau dies vor. Über drei solcher Fälle von Rhabdomyolyse nach Indoorcycling haben Mediziner um Maureen Brogan vom Westchester Medical Center in Valhalla, New York, im American Journal of Medicine berichtet.

- Fall 1: Eine 33-jährige Frau kommt mit Schmerzen und Schwellungen in beiden Oberschenkeln sowie Muskelschwäche in die Klinik. Vier Tage zuvor hatte sie intensiv auf einem Indoor-Rad trainiert. Nach 15 Minuten musste sie absteigen, weil sie benommen wurde und sich übergeben musste. Die Blutuntersuchung ergibt folgende Werte: Kreatinin $0,5 \mathrm{mg} / \mathrm{dl}$ (Normwert für Frauen bis $1,0 \mathrm{mg} / \mathrm{dl})$, dAspartat-Aminotransferase (AST) $1.092 \mathrm{U} / \mathrm{l}(<35)$, AlaninAminotransferase (ALT) 282 U/l $(<35)$ und Kreatinkinase (CK) $60.000 \mathrm{U} / 1$ $(<145)$.

- Fall 2: Ein 20-jähriger Mann, anamnestisch mit Morbus Meulengracht belastet, stellt sich drei Tage nach einem anstrengenden Ergometertraining mit Schmerzen in den Oberschenkeln vor, unfähig, die Beine zu heben. Die Schenkel sind geschwollen. Das Kreatinin ist normal, AST und ALT sind massiv erhöht. Der Spitzenwert der CK beträgt 132.832 U/l (Norm für Männer < 170).

- Fall 3: Eine 33-Jährige erscheint zwei Tage nach kräftezehrendem Spinning mit schmerzenden, geschwollenen Ober- schenkeln. Die Urinausscheidung ist vermindert, der Patientin ist übel und sie muss sich übergeben. ALT, AST und CK sind massiv erhöht. Vor allem aber hat sie einen Kreatininwert von $6,8 \mathrm{mg} /$ dl. Trotz aggressiver Hydratation mit isotonischer Kochsalzlösung rutscht sie in ein dialysepflichtiges akutes Nierenversagen. Die Nierenbiospie bestätigt ein akutes myoglobinurisches Nierenversagen auf der Basis einer Rhabdomyolyse. Fünfeinhalb Wochen später liegt das Kreatinin bei $0,7 \mathrm{mg} / \mathrm{dl}$, auch die CK hat sich normalisiert.
Fazit: Fälle von Rhabdomyolyse ereignen sich nicht nur nach intensivem Indoorcycling. Allerdings sind hier bereits einige Fälle, nicht nur die drei hier Genannten, aktenkundig. Brogan und Kollegen mahnen daher Anfänger, es langsam angehen zu lassen, die Sicherheitsbestimmungen einzuhalten, die Belastung graduell $\mathrm{zu}$ steigern und ausreichend zu trinken. Außerdem sollten Intensiv-Cycler über das Risiko und die Symptome einer Rhabdomyolyse informiert werden - und über die dringende Notwendigkeit, im Fall von Beschwerden umgehend einen Arzt aufzusuchen.

Dr. Robert Bublak

Brogan M et al. Freebie Rhabdomyolysis: A Public Health Concern. Spin Class-Induced Rhabdomyolysis. Am J Med 2017; 130: 484-7.
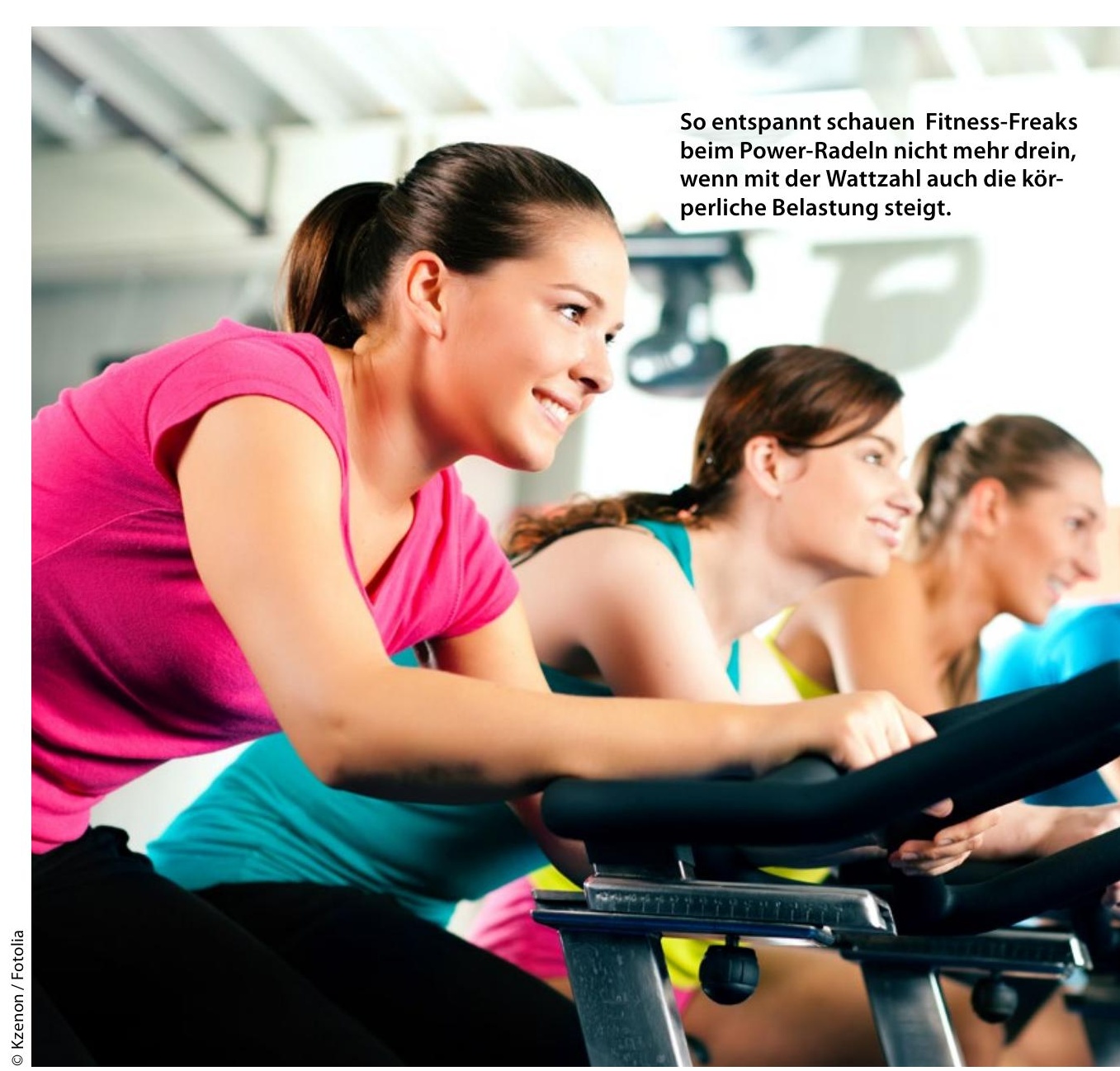